\title{
Bochner-Riesz operators in grand lebesgue spaces
}

\author{
Maria Rosaria Formica ${ }^{1}$ (I) $\cdot$ Eugeny Ostrovsky ${ }^{2} \cdot$ Leonid Sirota $^{2}$
}

Received: 8 February 2021 / Revised: 15 May 2021 / Accepted: 24 May 2021 / Published online: 31 May 2021 (c) The Author(s) 2021

\section{Abstract}

We provide the conditions for the boundedness of the Bochner-Riesz operator acting between two different Grand Lebesgue Spaces. Moreover we obtain a lower estimate for the constant appearing in the Lebesgue-Riesz norm estimation of the Bochner-Riesz operator and we investigate the convergence of the Bochner-Riesz approximation in Lebesgue-Riesz spaces.

Keywords Bochner-Riesz operator - Lebesgue-Riesz spaces · Grand Lebesgue spaces · Fourier transform · convolution · Beckner-Brascamp-Lieb inequality · Gaussian density · Dominated convergence theorem · Bessel's and Gamma functions · Modulus of continuity

Mathematics Subject Classification 46E30 - 44A35 - 42B15

\section{Introduction}

The well-known (pseudo-differential) Bochner-Riesz linear operator $B_{R}^{\alpha}[f]$, acting on measurable functions $f: \mathbb{R}^{n} \rightarrow \mathbb{R}(n>1)$, is defined as follows

$$
B_{R}^{\alpha}[f](t) \stackrel{\text { def }}{=} \int_{\mathbb{R}^{n}} e^{-i(t, y)}\left(1-\frac{|y|^{2}}{R^{2}}\right)_{+}^{\alpha} \tilde{f}(y) d y,
$$

$凶 \quad$ Maria Rosaria Formica

mara.formica@uniparthenope.it

Eugeny Ostrovsky

eugostrovsky@list.ru

Leonid Sirota

sirota3@bezeqint.net

1 Università degli Studi di Napoli "Parthenope", via Generale Parisi 13, 80132 Palazzo

Pacanowsky, Napoli, Italy

2 Department of Mathematics and Statistics, Bar-Ilan University, 52900 Ramat Gan, Israel

Birkhäuser 
where

$$
R=\text { const }>0, \quad \alpha=\text { const }, \quad z_{+}=\max (z, 0), \quad z \in \mathbb{R},
$$

$(x, y)$ stands for the scalar (inner) product of two vectors $x, y \in \mathbb{R}^{n},|y|^{2}:=(y, y)$ and $\tilde{f}$ denotes, as ordinary, the Fourier transform

$$
\tilde{f}(y)=\int_{\mathbb{R}^{n}} e^{i(x, y)} f(x) d x .
$$

The applications of these operators are described in particular in Functional Analysis (see [10,23]) and in statistics of random processes and field (see [5,41]).

The classical Lebesgue-Riesz norm $\|f\|_{p}, \quad p \in[1, \infty]$, for the function $f$, is denoted by

$$
\begin{aligned}
\|f\|_{p} & :=\left[\int_{\mathbb{R}^{n}}|f(x)|^{p} d x\right]^{1 / p}, \quad 1 \leq p<\infty, \\
\|f\|_{\infty}: & =\operatorname{ess}_{\sup } \operatorname{si\mathbb {R}}^{n}|f(x)|, \quad p=\infty
\end{aligned}
$$

and the corresponding Banach space is, as usually,

$$
L_{p}=L_{p}\left(\mathbb{R}^{n}\right)=\left\{f:\|f\|_{p}<\infty\right\} .
$$

Denote, for an arbitrary linear or quasi linear operator $U$, acting from $L_{p}$ to $L_{q}, p, q \geq 1$, its norm

$$
\|U\|_{p, q}=\|U\|_{p \rightarrow q}:=\sup _{0 \neq f \in L_{p}}\left[\frac{\|U f\|_{q}}{\|f\|_{p}}\right] .
$$

Of course, the operator $U$ is bounded as the operator acting from the space $L_{p}$ into the space $L_{q}$ iff $\|U\|_{p, q}<\infty$.

More generally, for the operator $U$ acting from some Banach space $F$ equipped with the norm $\|\cdot\|_{F}$ into another (in the general case) Banach space $D$, having the norm $\|\cdot\|_{D}$, we denote as usually

$$
\|U\|_{F \rightarrow D}:=\sup _{0 \neq f \in F}\left[\frac{\|U f\|_{D}}{\|f\|_{F}}\right] .
$$

There exists a huge numbers of works devoted to the $p, q$ estimates for BochnerRiesz operators $B_{R}^{\alpha}$, as a rule for the case $q=p$, see e.g. [23, chapter5], [9,10,13,29, $35,42,43]$, etc. The boundedness of these operators in Morrey-Lorentz spaces and in $L^{p}$ spaces with variable exponent has been investigated in [25] and [8], respectively.

Our aim, in this paper, is to extend some results contained in the above mentioned works concerning upper estimates for the norm of the Bochner-Riesz operator, in the case of different Lebesgue-Riesz spaces (Sect. 2) and to extend these estimates to the so-called Grand Lebesgue Spaces (GLS), see Sect. 3. 
We deduce also a non trivial quantitative lower estimate for the coefficient in the Lebesgue-Riesz norm estimation for the Bochner-Riesz operator (Sect. 4) and we study the convergence of the Bochner-Riesz approximation in Lebesgue-Riesz spaces (Sect. 5).

\section{Norm estimates for the Bochner-Riesz operator acting between different Lebesgue-Riesz spaces}

We clarify slightly the known results about the Lebesgue-Riesz $p, r$ norms for the operators $B_{R}^{\alpha}$ defined in (1.1), see e.g. [10,12,23,29,43], ecc.

It is important to observe that the Bochner-Riesz operator $B_{R}^{\alpha}$ may be rewritten as a convolution, namely

$$
B_{R}^{\alpha}[f](t)=f * K_{\lambda}^{R}(t)=\int_{\mathbb{R}^{n}} f(t-s) K_{\lambda}^{R}(s) d s,
$$

where $R=$ const $>0$,

$$
\begin{aligned}
K_{\lambda}^{R}(z) & =C(\alpha) R^{n} \frac{J_{\lambda}(R|z|)}{|R z|^{\lambda}}, \quad \lambda=\alpha+\frac{n}{2}, \\
C(\alpha) & :=\frac{1}{\Gamma(\alpha+1)}, \quad \alpha>-1,
\end{aligned}
$$

$\Gamma(\cdot)$ is the Gamma-function, $J_{\lambda}(\cdot)$ is the Bessel function of order $\lambda$ and $|z|=\sqrt{(z, z)}$.

Notice that, under our restriction on $\alpha$,

$$
\int_{\mathbb{R}^{n}} K_{\lambda}^{R}(t) d t=1
$$

Evidently,

$$
K_{\lambda}^{R}(z)=R^{n} K_{\lambda}(R z)
$$

where in turn

$$
K_{\lambda}(z) \stackrel{\text { def }}{=} K_{\lambda}^{1}(z)=C(\alpha) \frac{J_{\lambda}(|z|)}{|z|^{\lambda}},
$$

see, e.g., [10, pp. 171-172].

Note that, from the identity (2.3), it follows

$$
\left\|K_{\lambda}^{R}(z)\right\|_{q}=R^{n-n / q}\left\|K_{\lambda}\right\|_{q}, \quad q \geq 1,
$$

as long as $R>0$.

Assume here and in the sequel

$$
-1<\alpha \leq \alpha_{0}, \quad \alpha_{0}:=\frac{n-1}{2},
$$


and introduce the value

$$
q_{0}:=\frac{n}{(n+1) / 2+\alpha}
$$

so that

$$
q_{0} \geq 1 \Leftrightarrow \frac{-(n+1)}{2}<\alpha \leq \alpha_{0},
$$

where the inequality on the left hand side is true due to the restriction $\alpha>-1$.

Moreover, using the well-known results about the behavior of the Bessel functions (see, e.g., [10, p. 172]),

$$
K_{\lambda}(z) \asymp|z|^{-\left(\frac{n+1}{2}+\alpha\right)}, \quad \text { as }|z| \rightarrow \infty,
$$

it is easy to estimate

$$
\left\|K_{\lambda}\right\|_{q} \asymp\left(q-q_{0}\right)^{-1 / q}, \quad q>q_{0},
$$

and $\left\|K_{\lambda}\right\|_{q}=\infty$ otherwise (see also, e.g., [23, pp. 339-341]).

Correspondingly,

$$
\left\|K_{\lambda}^{R}\right\|_{q} \asymp R^{n-n / q}\left(q-q_{0}\right)^{-1 / q}, \quad q>q_{0} .
$$

Furthermore, we recall the Beckner-Brascamp-Lieb-Young inequality for the convolution (see $[3,4])$

$$
\|f * g\|_{r} \leq\left(\frac{C_{p} C_{q}}{C_{r}}\right)^{n}\|f\|_{p}\|g\|_{q}, \quad 1+1 / r=1 / p+1 / q,
$$

where $p, q, r \geq 1$ and

$$
C_{m}=\left(\frac{m^{1 / m}}{\left(m^{\prime}\right)^{1 / m^{\prime}}}\right)^{\frac{1}{2}}, \quad m^{\prime}=m /(m-1) .
$$

We point out that the constant $C_{m}$ defined above, and considered in (2.10) for $m=$ $p, q, r$, does not blow up when $m \rightarrow 1$ (which implies $m^{\prime} \rightarrow \infty$ ), in fact $C_{m} \rightarrow 1$ in this case. Therefore $p, q, r$ are allowed to be 1 . Note that

$$
\frac{C_{p} C_{q}}{C_{r}} \leq 1
$$

Recently in [22] has been given a generalization of the convolution inequality in the context of the Grand Lebesgue Spaces (see Sect. 3 for the definition), built on a unimodular locally compact topological group.

The estimate (2.10) is essentially non-improbable. Indeed, the equality in (2.10) is attained iff both functions $f, g$ are proportional to Gaussian densities, namely there exists positive constants $c_{1}, c_{2}, c_{3}, c_{4}$ such that

$$
f(x)=c_{1} \exp \left(-c_{2}|x|^{2}\right), \quad g(x)=c_{3} \exp \left(-c_{4}|x|^{2}\right), \quad x \in \mathbb{R}^{n},
$$


where $|x|=\sqrt{(x, x)}$ is the euclidean norm. Then the convolution $f * g$ is also Gaussian and

$$
\|f * g\|_{r}=\left(\frac{C_{p} C_{q}}{C_{r}}\right)^{n}\|f\|_{p}\|g\|_{q}, \quad 1 / p+1 / q=1+1 / r, \quad p, q, r>1 .
$$

The following result about the boundedness of the Bochner-Riesz operator, acting between different Lebesgue-Riesz spaces, holds.

Theorem 2.1 Let $p>1$ and $f \in L_{p}\left(\mathbb{R}^{n}\right)$. Let $\alpha$ be a constant such that

$$
-1<\alpha \leq \alpha_{0}:=\frac{n-1}{2}
$$

and

$$
q_{0}=\frac{n}{(n+1) / 2+\alpha} .
$$

Let $q, r \geq 1$ such that $1+1 / r=1 / q+1 / p$ and assume $q>q_{0}, r>r_{0}, p \leq p_{0}$, where

$$
\begin{aligned}
& r_{0}=r_{0}(\alpha, n ; p):=\frac{p q_{0}}{p+q_{0}-p q_{0}}, \\
& p_{0}=q_{0}^{\prime}=\left(1-1 / q_{0}\right)^{-1}
\end{aligned}
$$

Define, for $R=$ const $>0$,

$$
W(\alpha, n, R ; p, r) \stackrel{\text { def }}{=} R^{n(1 / p-1 / r)} \cdot\left[q(p, r)-q_{0}(\alpha, n)\right]^{1 / p-1-1 / r} .
$$

Then the Bochner-Riesz operator satisfies

$$
\left\|B_{R}^{\alpha}[f]\right\|_{r} \leq C(\alpha, R) \cdot W(\alpha, n, R ; p, r) \cdot\|f\|_{p}
$$

Proof If $f \in L_{p}$ for some value $p>1$, then by (2.10) it follows

$$
\left\|B_{R}^{\alpha}[f]\right\|_{r} \leq\left\|K_{\lambda}^{R}\right\|_{q}\|f\|_{p}
$$

where $1+1 / r=1 / q+1 / p$ and $p, q, r \geq 1, q>q_{0}$. On the other words,

$$
q=q(p, r) \stackrel{\text { def }}{=} \frac{p r}{p r+p-r}, \quad q>q_{0}, \quad r>p
$$

Moreover it is easy to verify that $r_{0}$, defined in (2.15), is such that $r_{0}>p$. Under our restrictions $W(\alpha, n, R ; p, r)$ is finite and positive.

So we conclude that estimate (2.18) holds. 


\section{Main result: boundedness of Bochner-Riesz operators in Grand Lebesgue Spaces (GLS)}

We recall here, for reader convenience, some known definitions and facts from the theory of Grand Lebesgue Spaces (GLS).

Definition 3.1 Let $\psi=\psi(p), p \in(a, b), a, b=$ const, $1 \leq a<b \leq \infty$, be a positive measurable numerical valued function, such that

$$
\inf _{p \in(a, b)} \psi(p)>0
$$

The (Banach) Grand Lebesgue Space $G \psi=G \psi(a, b)$ consists of all the real (or complex) numerical valued measurable functions $f: \mathbb{R}^{n} \rightarrow \mathbb{R}$ having finite norm, defined by

$$
\|f\|_{G \psi}:=\sup _{p \in(a, b)}\left[\frac{\|f\|_{p}}{\psi(p)}\right] .
$$

We agree to write $G \psi$ in the case when $a=1$ and $b=\infty$.

The function $\psi$ is named generating function for the space $G \psi$ and we denote by $\{\psi(\cdot)\}$ the set of all such functions.

For instance

$$
\psi(p):=p^{1 / m}, \quad p \in[1, \infty), \quad m>0,
$$

or

$$
\psi(p):=(p-a)^{-\alpha}(b-p)^{-\beta}, \quad p \in(a, b), \quad 1 \leq a<b<\infty, \quad \alpha, \beta \geq 0,
$$

are generating functions.

If

$$
\psi(p)=1, \quad p=r ; \quad \psi(p)=+\infty, \quad p \neq r, \quad r \in[1, \infty)
$$

where $C / \infty:=0, C \in \mathbb{R}$ (extremal case), then the corresponding $G \psi$ space coincides with the classical Lebesgue-Riesz space $L_{r}=L_{r}\left(\mathbb{R}^{d}\right)$.

The Grand Lebesgue Spaces and several generalizations of them have been widely investigated, mainly in the case of GLS on sets of finite measure, (see, e.g., [6,11,14, $20,26,27,34,36]$, etc). They play an important role in the theory of Partial Differential Equations (PDEs) (see, e.g., [2,15,17,24], etc.), in interpolation theory (see, e.g., [16, $19])$, in the theory of Probability $([21,38,40])$, in Statistics [36, chapter 5], in theory of random fields [30,39], in Functional Analysis [36,37,39] and so one.

These spaces are rearrangement invariant (r.i.) Banach functional (complete) spaces; their fundamental functions have been considered in [39]. They do not coincide, in the general case, with the classical Banach rearrangement functional spaces: Orlicz, Lorentz, Marcinkiewicz etc., see [34,37]. The belonging of a function $f: \mathbb{R}^{n} \rightarrow \mathbb{R}$ to some $G \psi$ space is closely related with its tail function behavior as $t \rightarrow 0+$ as well as when $t \rightarrow \infty$, see [30,32]. 
The Grand Lebesgue Spaces can be considered not only on the Euclidean space $\mathbb{R}^{n}$ equipped with the Lebesgue measure, but also on an arbitrary measurable space with sigma-finite non-trivial measure.

In the following Theorem we investigate the boundedness of the Bochner-Riesz operator acting from some Grand Lebesgue Space $G \psi=G \psi(a, b)$ into another one $G \nu$. We will consider the same restrictions and quantities defined in Theorem 2.1.

Theorem 3.1 Let $1 \leq a<b \leq \infty$ and $f \in G \psi(a, b)$. Let $\alpha$ be a constant such that

$$
-1<\alpha \leq \alpha_{0}:=\frac{n-1}{2}, \quad n>1
$$

Let $p>1, q, r \geq 1, p_{0}, q_{0}, r_{0}$ and $W(\alpha, n, R ; p, r)$ defined as in Theorem 2.1.

Denote

$$
s=s(\alpha, n ; r):=\min \left\{b, \frac{r q_{0}}{r q_{0}+q_{0}-r}\right\},
$$

and

$$
d:=r_{0}(\alpha, n ; b)=\frac{b q_{0}}{q_{0}+b-b q_{0}} .
$$

For $r \in(d, \infty)$ let $v(r)$ be the following generating function

$$
v(r)=v[\psi](r)=v[\psi](\alpha, n, R ; r):=\inf _{p \in(a, s)}[W(\alpha, n, R ; p, r) \psi(p)] .
$$

Then

$$
\left\|B_{R}^{\alpha}[f]\right\|_{G v} \leq C(\alpha, n, R)\|f\|_{G \psi} .
$$

Proof The proof is simple and alike as the one in [40]. First we observe that $v(r)$ is finite. One can assume, without loss of generality, $\|f\|_{G \psi}=1$, then $\|f\|_{p} \leq$ $\psi(p), p \in(a, b)$. Applying the inequality (2.18) we have

$$
\left\|B_{R}^{\alpha}[f]\right\|_{r} \leq C(\alpha, n, R) \cdot W(\alpha, n, R ; p, r) \cdot \psi(p)
$$

Taking the minimum over $p$ subject to our limitations, we get

$$
\begin{aligned}
\left\|B_{R}^{\alpha}[f]\right\|_{r} & \leq C(\alpha, n, R) \cdot \inf _{p \in(a, s)}[W(\alpha, R ; p, r) \cdot \psi(p)] \\
& =C(\alpha, n, R) v(r)=C(\alpha, n, R) v(r)\|f\|_{G \psi},
\end{aligned}
$$

which is quite equivalent to our claim in (3.6).

Remark 3.1 Let us give the following generalization of Theorem 3.1 Let $\zeta=\zeta(r), r \in$ $(d, \infty)$, be some another generating function from the set $G \Psi[d, \infty]$, such that a 
new function

$$
\kappa(r) \stackrel{\text { def }}{=} \sup _{p \in(a, s)}\left\{\frac{W(\alpha, R ; p, r) \psi(p)}{\zeta(r)}\right\}
$$

also belongs to this set. We deduce from (3.7)

$$
\frac{\left\|B_{R}^{\alpha}[f]\right\|_{r}}{\zeta(r)} \leq C(\alpha, n, R) \cdot \frac{W(\alpha, n, R ; p, r) \cdot \psi(p)}{\zeta(r)} \cdot\|f\| G \psi,
$$

hence

$$
\frac{\left\|B_{R}^{\alpha}[f]\right\|_{r}}{\zeta(r)} \leq C(\alpha, n, R) \kappa(r)\|f\| G \psi
$$

Dividing on $\kappa(r)$ and taking the supremum over $r$, if we denote $\theta(r)=\zeta(r) \kappa(r)$, we have

$$
\left\|B_{R}^{\alpha}[f]\right\|_{G \theta} \leq C(\alpha, n, R)\|f\|_{G \psi} .
$$

\section{Lower bound for the coefficient in the Lebesgue-Riesz norm estimate for the Bochner-Riesz operator}

Let $p, r>1, n>1$ and let us introduce the following variable

$$
Q_{n}(p, r) \stackrel{\text { def }}{=} \sup _{\alpha>0} \sup _{R>0} W(\alpha, n, R ; p, r)
$$

where $W(\alpha, n, R ; p, r)$ is defined in Sect. 2. Our target in this Section is a lower bound for the above variable.

Theorem 4.1 Let $p, r>1, n>1$ and

$$
\Theta(n, p):=(2 \pi)^{n(1-p) / 2 p} p^{-n / 2 p} .
$$

Then

$$
Q_{n}(p, r) \geq \Theta\left(n, \frac{p r}{p r+p-r}\right), \quad r>p .
$$

Remark 4.1 The possible case when $Q_{n}(p, r)=+\infty$ can not be excluded.

Proof We will apply equality (2.12), in which we choose the ordinary Gaussian density

$$
f_{0}(x):=(2 \pi)^{-n / 2} \exp \left(-|x|^{2} / 2\right), \quad x \in \mathbb{R}^{n},
$$

and take $\alpha=R^{2} / 2$. Obviously

$$
Q_{n}(p, r) \geq \lim _{R \rightarrow \infty} W\left(R^{2} / 2, n, R ; p, r\right)
$$


We have

$$
B_{R}^{R^{2}}[f](t)=\int_{\mathbb{R}^{n}} e^{-i(t, y)}\left(1-\frac{|y|^{2}}{R^{2}}\right)^{R^{2} / 2} I(|y|<R) \tilde{f}(y) d y,
$$

where $I(A)$ denotes the indicator function of the (measurable) set $A, A \subset \mathbb{R}^{n}$. Therefore, as $R \rightarrow \infty$,

$$
B_{R}^{R^{2}}[f](t) \rightarrow \int_{\mathbb{R}^{n}} e^{-i(t, y)} \exp \left\{-|y|^{2} / 2\right\} \tilde{f}(y) d y=[\sqrt{2 \pi}]^{n}\left[f * f_{0}\right](t),
$$

by virtue of dominated convergence theorem.

If we take $f=f_{0}$, then in (4.2) the convolution of two Gaussian densities appears. It remains to apply the relation (2.12); we omit some simple calculations.

\section{Convergence of Bochner-Riesz operators}

We investigate here the convergence, as $R \rightarrow \infty$, of the family of Bochner-Riesz approximations $B_{R}^{\alpha}[f]$ to the source function $f$ in the Lebesgue-Riesz norm $L_{p}\left(\mathbb{R}^{n}\right), p \in(1, \infty)$, in addition to the similar results in [13,23,29], etc.

For any function $f \in L_{p}\left(\mathbb{R}^{n}\right)$, its modulus of $L_{p}$ continuity is defined alike as in approximation theory [1, chapter $\mathrm{V}]$ :

$$
\omega_{p}[f](\delta) \stackrel{\text { def }}{=} \sup _{h:|h| \leq \delta}\left\|T_{h}[f]-f\right\|_{p}, \quad \delta \geq 0
$$

where $T_{h}[f]$ denotes the shift operator

$$
T_{h}[f](t)=f(t-h), \quad t, h \in \mathbb{R}^{n} .
$$

Obviously, $\omega_{p}[f](\delta) \leq 2\|f\|_{p}$ and

$$
\lim _{\delta \rightarrow 0+} \omega_{p}[f](\delta)=0, \quad f \in L_{p}
$$

Theorem 5.1 Let $p \in(1, \infty), \alpha \leq \alpha_{0}=(n-1) / 2$ and $f \in L_{p}\left(\mathbb{R}^{n}\right)$. Then

$$
\lim _{R \rightarrow \infty}\left\|B_{R}^{\alpha}[f]-f\right\|_{p}=0
$$

Proof The difference $\Delta_{R}[f](t)=B_{R}^{\alpha}[f]-f$ has the form

$$
\Delta_{R}[f](t)=\int_{\mathbb{R}^{n}}\left\{f\left(t-\frac{v}{R}\right)-f(t)\right\} K_{\lambda}(|v|) d v
$$


We apply now the triangle inequality for the $L_{p}$ norm in the integral form

$$
\begin{aligned}
\left\|\Delta_{R}[f]\right\|_{p} & \leq \int_{\mathbb{R}^{n}}\left\|T_{|v| / R}[f]-f\right\|_{p}\left|K_{\lambda}(|v|)\right| d v \\
& \leq \int_{\mathbb{R}^{n}} \omega_{p}[f](|v| / R)\left|K_{\lambda}(|v|)\right| d v .
\end{aligned}
$$

Note that, under the above conditions,

$$
\int_{\mathbb{R}^{n}}\left|K_{\lambda}(|v|)\right| d v<\infty
$$

so that (5.2) follows again from the dominated convergence theorem.

Remark 5.1 As a slight consequence, under the above conditions, if $f \in L_{p}\left(\mathbb{R}^{n}\right)$, then

$$
\left\|B_{R}^{\alpha}[f]\right\|\left\|_{p} \leq\right\| f \|_{p}+\int_{\mathbb{R}^{n}} \omega_{p}[f](|v| / R)\left|K_{\lambda}(|v|)\right| d v
$$

and, consequently,

$$
\forall f \in L_{p}\left(\mathbb{R}^{n}\right) \Rightarrow \sup _{R \geq 1}\left\|B_{R}^{\alpha}[f]\right\|_{p} \leq 3\|f\|_{p} .
$$

The case $p=\infty$ requires a separate consideration. Introduce the Banach space $C_{0}\left(\mathbb{R}^{n}\right)$ as a collection of all bounded and uniformly continuous functions $f: \mathbb{R}^{n} \rightarrow$ $\mathbb{R}$, equipped with the ordinary norm,

$$
\|f\|_{\infty} \stackrel{\text { def }}{=} \sup _{t \in \mathbb{R}^{n}}|f(t)|
$$

As above

$$
\omega_{\infty}[f](\delta) \stackrel{\text { def }}{=} \sup _{h:|h| \leq \delta}\left\|T_{h}[f]-f\right\|_{\infty}, \quad \delta \geq 0 .
$$

Evidently, $\omega_{\infty}[f](\delta) \leq 2\|f\|_{\infty}$ and

$$
\lim _{\delta \rightarrow 0+} \omega_{\infty}[f](\delta)=0, \quad f \in L_{p}
$$

The assertion of Theorem 5.1 under the same conditions remains true in the case $p=\infty$.

Theorem 5.2 Under the same conditions of Theorem 5.1, for any function $f: \mathbb{R}^{n} \rightarrow$ $\mathbb{R}$ from the space $C_{0}\left(\mathbb{R}^{n}\right)$, its Bochner-Riesz approximation $B_{R}^{\alpha}$ converges uniformly to the source function $f$, that is

$$
\lim _{R \rightarrow \infty}\left\|B_{R}^{\alpha}[f]-f\right\|_{\infty}=0
$$


Proof The proof is the same as in Theorem 5.1 and may be omitted.

Remark 5.2 As before, if $f \in C_{0}\left(\mathbb{R}^{n}\right)$, then

$$
\left\|B_{R}^{\alpha}[f]\right\|_{\infty} \leq\|f\|_{\infty}+\int_{\mathbb{R}^{n}} \omega_{\infty}[f](|v| / R)\left|K_{\lambda}(|v|)\right| d v
$$

and moreover

$$
\forall f \in C_{0}\left(\mathbb{R}^{n}\right) \Rightarrow \sup _{R \geq 1}\left\|B_{R}^{\alpha}[f]\right\|_{\infty} \leq 3\|f\|_{\infty}
$$

\section{Concluding remarks}

A. In our opinion, the method described in this paper may be essentially generalized to more operators of convolutions type, linear or not. See some preliminary results [34].

B. The study of the maximal Bochner-Riesz operator $B_{*}^{\alpha}$, defined by

$$
B_{*}^{\alpha}[f](t)=\sup _{R>0}\left|B_{R}^{\alpha}[f](t)\right|
$$

is closely related to the pointwise convergence of the associated Bochner-Riesz operator $B_{R}^{\alpha}[f]$ as $R \rightarrow \infty$, for any $f \in L_{p}\left(\mathbb{R}^{n}\right), p \in(1, \infty)$, see e. g. [7,33,44].

It is interesting to generalize the estimates obtained in the previous Sections to the maximal operator associated to the Bochner-Riesz one considered here, in the spirit of the works $[18,28]$, and so one:

$$
\left\|\sup _{R \geq 1} B_{R}^{\alpha}[f]\right\|_{p} \leq M(\alpha, n ; p) \cdot\|f\|_{p}, \quad p \geq p_{0}
$$

or

$$
\left\|\sup _{R \geq 1} B_{R}^{\alpha}[f]\right\|_{r} \leq M(\alpha, n ; p, r) \cdot\|f\|_{p}, \quad p \geq p_{0}, \quad r=r(p)
$$

in order to obtain the GLS estimate for the Bochner-Riesz maximal operator of the form

$$
\left\|\sup _{R \geq 1} B_{R}^{\alpha}[f]\right\|_{G \zeta} \leq L(\alpha, n ; \zeta, \psi) \cdot\|f\|_{G \psi}
$$

for some generating functions $\psi, \zeta$. 
C. It is not hard to generalize the estimates obtained here in the case when the kernels are of the form

$$
\phi(t):=\prod_{s=1}^{k}\left(1-t^{\alpha(s)}\right)_{+}^{m(s)},
$$

instead of the the kernel $\left(1-t^{2}\right)_{+}^{\alpha}$, considered here. Necessary estimates for this purpose for the Fourier transform $F[\phi](y)$ of these functions may be found in $[45,46]$.

Acknowledgements The first author has been partially supported by the Gruppo Nazionale per l'Analisi Matematica, la Probabilità e le loro Applicazioni (GNAMPA) of the Istituto Nazionale di Alta Matematica (INdAM) and by Università degli Studi di Napoli Parthenope through the project "sostegno alla Ricerca individuale".

Funding Open access funding provided by Università Parthenope di Napoli within the CRUI-CARE Agreement.

Open Access This article is licensed under a Creative Commons Attribution 4.0 International License, which permits use, sharing, adaptation, distribution and reproduction in any medium or format, as long as you give appropriate credit to the original author(s) and the source, provide a link to the Creative Commons licence, and indicate if changes were made. The images or other third party material in this article are included in the article's Creative Commons licence, unless indicated otherwise in a credit line to the material. If material is not included in the article's Creative Commons licence and your intended use is not permitted by statutory regulation or exceeds the permitted use, you will need to obtain permission directly from the copyright holder. To view a copy of this licence, visit http://creativecommons.org/licenses/by/4.0/.

\section{References}

1. Achiezer, N.I.: Theory of Approximation. F. Ungar, Translated from Russian (1956)

2. Ahmed, I., Fiorenza, A., Formica, M.R., Gogatishvili, A., Rakotoson, J.M.: Some new results related to Lorentz G-Gamma spaces and interpolation. J. Math. Anal. Appl. 483(2), 123623 (2020)

3. Beckner, W.: Inequalities in Fourier analysis. Ann. Math. 102, 159-182 (1975)

4. Brascamp, H.J., Lieb, E.H.: Best constants in Young's inequality, its converse, and its generalization to more than three functions. Adv. Math. 20(2), 151-173 (1976)

5. Buldygin, V.V., Ilarionov, E.V.: A problem in the statistics of random fields. (Russian) Probabilistic infinite-dimensional analysis, pp. 6-14, 123, Akad. Nauk Ukrain. SSR, Inst. Mat., Kiev, (1981)

6. Capone, C., Formica, M.R., Giova, R.: Grand Lebesgue spaces with respect to measurable functions. Nonlinear Anal. 85, 125-131 (2013)

7. Carbery, A.: The boundedness of the maximal Bochner-Riesz operator on $L_{4}\left(\mathbb{R}^{2}\right)$. Duke Math. J. 50(2), 409-416 (1983)

8. Chen, D.: Multilinear integral operators on $L^{p}$ spaces with variable exponent. Integral Transf. Spec. Funct. 30(12), 962-977 (2019)

9. Córdoba, A.: A note on Bochner-Riesz operators. Duke Math. J. 46(3), 505-511 (1979)

10. Duoandikoetxea, J.: Fourier analysis. Graduate Studies in Math., vol. 29. American Mathematical Society, Providence, RI. Translated and revised from the 1995 Spanish original by David Cruz-Uribe (2001)

11. Ermakov, S.V., Ostrovsky, E.I.: Continuity Conditions, Exponential Estimates, and the Central Limit Theorem for Random Fields. VINITY, Moscow (1986) ((in Russian))

12. Fefferman, C.: Inequalities for strongly singular convolution operators. Acta Math. 124, 9-36 (1970)

13. Fefferman, C.: A note on spherical summation multipliers. Israel J. Math. 15, 44-52 (1973)

14. Fiorenza, A.: Duality and reflexivity in grand Lebesgue spaces. Collect. Math. 51(2), 131-148 (2000) 
15. Fiorenza, A., Formica, M.R., Gogatishvili, A.: On grand and small Lebesgue and Sobolev spaces and some applications to PDE's. Differ. Equ. Appl. 10(1), 21-46 (2018)

16. Fiorenza, A., Formica, M.R., Gogatishvili, A., Kopaliani, T., Rakotoson, J.M.: Characterization of interpolation between grand, small or classical Lebesgue spaces. Nonlinear Anal. 177, 422-453 (2018)

17. Fiorenza, A., Formica, M.R., Rakotoson, J.M.: Pointwise estimates for $G \Gamma$-functions and applications. Differ. Equ. 30(11-12), 809-824 (2017)

18. Fiorenza, A., Gupta, B., Jain, P.: The maximal theorem for weighted grand Lebesgue spaces. Studia Math. 188(2), 123-133 (2008)

19. Fiorenza, A., Karadzhov, G.E.: Grand and small Lebesgue spaces and their analogs. Z. Anal. Anwend. 23(4), 657-681 (2004)

20. Formica, M.R., Giova, R.: Boyd indices in generalized grand Lebesgue spaces and applications. Mediterr. J. Math. 12(3), 987-995 (2015)

21. Formica, M.R., Kozachenko, Y.V., Ostrovsky, E., Sirota, L.: Exponential tail estimates in the law of ordinary logarithm (LOL) for triangular arrays of random variables. Lith. Math. J. 60, 330-358 (2020)

22. Formica, M.R., Ostrovsky, E., Sirota, L.: Grand Lebesgue Spaces are really Banach algebras relative to the convolution on unimodular locally compact groups equipped with Haar measure, to appear on Math. Nachr

23. Grafakos, L.: Modern Fourier Analysis, 3rd edn. Graduate Texts in Mathematics, vol. 250. Springer, New York (2014)

24. Greco, L., Iwaniec, T., Sbordone, C.: Inverting the p-harmonic operator. Manuscripta Math. 92(2), 249-258 (1997)

25. Guliyev, V.S., Aykol, C., Kucukaslan, A., Serbetci, A.: Maximal operator and Calderon-Zygmund operators in local Morrey-Lorentz spaces. Integral Transf. Spec. Funct. 27(11), 866-877 (2016)

26. Iwaniec, T., Sbordone, C.: On the integrability of the Jacobian under minimal hypotheses. Arch. Ration. Mech. Anal. 119(2), 129-143 (1992)

27. Iwaniec, T., Koskela, P., Onninen, J.: Mappings of finite distortion: monotonicity and continuity. Invent. Math. 144(3), 507-531 (2001)

28. Jain, P., Kumari, S.: On grand Lorentz spaces and the maximal operator. Georg. Math. J. 19(2), 235-246 (2012)

29. Karasev, D.N., Nogin, V.A.: $L_{p}-L_{q}$ estimates for the Bochner-Riesz operator of complex order. Z. Anal. Anwendungen 21(4), 915-929 (2002)

30. Kozachenko, Yu.V., Ostrovsky, E.I.: Banach Spaces of random variables of sub-Gaussian type, Teor. Veroyatn. Mat. Stat., Kiev, 32 (134) (1985), 42-53 (in Russian). English transl.: Theory Probab. Math. Stat., 32, 45-56 (1986)

31. Kozachenko, Y.V., Mlavets, Y.Y., Yurchenko, N.V.: Weak convergence of stochastic processes from spaces $F_{\psi}(\Omega)$. Stat. Optim. Inform. Comput. 6(2), 266-277 (2018)

32. Kozachenko, Yu.V., Ostrovsky, E., Sirota, L.: Relations between exponential tails, moments and moment generating functions for random variables and vectors (2017). arXiv:1701.01901v1 [math.FA]

33. Lee, S.: Improved bounds for Bochner-Riesz and maximal Bochner-Riesz operators. Duke Math. J. 122(1), 205-232 (2004)

34. Liflyand, E., Ostrovsky, E., Sirota, L.: Structural properties of bilateral grand Lebesque spaces. Turkish J. Math. 34(2), 207-219 (2010)

35. Lu, S., Yan, D.: Bochner-Riesz Means on Euclidean Spaces. World Scientific Publishing Co. Pte. Ltd., Hackensack (2013)

36. Ostrovsky, E.: Exponential Orlicz's spaces: new norms and applications (2004). arXiv:math/0406534

37. Ostrovsky, E., Sirota, L.: Some new rearrangement invariant spaces: theory and applications, v1, 29, (2006). arXiv:math.FA/0605732

38. Ostrovsky, E., Sirota, L.: Fourier Transforms in Exponential Rearrangement Invariant Spaces, v1 (2004). arXiv:math.FA/040639

39. Ostrovsky, E., Sirota, L.: Moment Banach spaces: theory and applications. HIAT J. Sci. Eng. C 4(1-2), 233-262 (2007)

40. Ostrovsky, E., Sirota, L.: Boundedness of operators in bilateral Grand Lebesgue Spaces, with exact and weakly exact constant calculation (2011). arXiv:1104.2963v1 [math.FA]

41. Ostrovsky, E., Tsykunova, S.Y: Asymptotic properties of the distribution of the maximum of a Gaussian nonstationary process that arises in covariance statistics, (Russian). Teor. Veroyatnost. i Primenen. 39(3), 641-649 (1994); translation in Theory Probab. Appl. 39 (1994), no. 3, 527-534 (1995) 
42. Stein, E.M.: Singular Integrals and Differentiability Properties of Functions. Princeton Mathematical Series, no. 30 Princeton University Press, Princeton (1970)

43. Stein, E.M.: Harmonic analysis: real-variable methods, orthogonality, and oscillatory integrals. With the assistance of Timothy S. Murphy. Princeton Mathematical Series, 43. Monographs in Harmonic Analysis, III. Princeton University Press, Princeton (1993)

44. Tao, T.: On the maximal Bochner-Riesz conjecture in the plane for $p<2$. AMS 354(5), 1947-1959 (2002)

45. Wang, J.: Generalized Bochner-Riesz means on spaces generated by smooth blocks. Comment. Math. Univ. Carolin. 44(3), 489-505 (2003)

46. Zheng, X.: Fourier transform for some functions with finite support. Chinese Sci. Bull. 29(21), 13311342 (1984)

Publisher's Note Springer Nature remains neutral with regard to jurisdictional claims in published maps and institutional affiliations. 